\title{
The Impact of Previous History of Bariatric Surgery on Outcome of COVID-19. A Nationwide Medico-Administrative French Study
}

\author{
Antonio lannelli ${ }^{1,2,3,4}$ (D) Samir Bouam ${ }^{5}$ - Anne-Sophie Schneck ${ }^{6}$ Sébastien Frey ${ }^{1,2} \cdot$ Kevin Zarca $^{7,8}$. \\ Jean Gugenheim ${ }^{1,2,3}$. Marco Alifano 9,10
}

Received: 14 July 2020 / Revised: 10 November 2020 / Accepted: 11 November 2020 / Published online: 18 November 2020

(C) Springer Science+Business Media, LLC, part of Springer Nature 2020

\begin{abstract}
Purpose To determine the risk of invasive mechanical ventilation and death in obese individuals with a history of bariatric surgery (BS) admitted for COVID-19.

Methods All obese inpatients recorded during a hospital stay by the French National Health Insurance were included, and their electronic health data were reviewed retrospectively. Patients who had undergone bariatric surgery comprised the BS group and patients with obesity but no history of BS served as controls. The primary outcome was COVID-19-related death and the secondary outcome was the need for invasive mechanical ventilation.

Results 4,248,253 obese individuals aged 15-75 years were included and followed for a mean observation time of 5.43 \pm 2.93 years. 8286 individuals with a previous diagnosis of obesity were admitted for COVID-19 between January 1 and May 15, 2020. Of these patients, 541 had a history of BS and 7745 did not. The need for invasive mechanical ventilation and death occurred in $7 \%$ and $3.5 \%$ of the BS group versus $15 \%$ and $14.2 \%$ of the control group, respectively. In logistic regression, the risk of invasive mechanical ventilation was independently associated with increasing age, male sex, and hypertension, and mortality was independently associated with increasing age, male sex, history of heart failure, cancer, and diabetes, whereas BS had an independent protective effect. Two random exact matching tests confirmed the protective effect of BS.

Conclusion This nationwide study showed that BS is independently associated with a reduced risk of death and invasive mechanical ventilation in obese individuals with COVID-19.
\end{abstract}

Keywords Obesity · Bariatric surgery · COVID-19 · Risk · Invasive mechanical ventilation

\section{Introduction}

Obesity, hypertension, cardiovascular disease, diabetes, chronic respiratory disease, and malignancy have all been

Antonio Iannelli

iannelli.a@chu-nice.fr

1 Université Côte d'Azur, Nice, France

2 Digestive Surgery and Liver Transplantation Unit, Archet2 Hospital, Centre Hospitalier Universitaire de Nice, Nice, France

3 Inserm, U1065, Team 8 "Hepatic Complications of Obesity and Alcohol", Nice, France

4 Service de Chirurgie Digestive et Centre de Transplantation Hépatique, Hôpital Archet 2, 151 Route Saint Antoine de Ginestière, BP 3079 Cedex3 Nice, France reported to be associated with a severe clinical course or even mortality in COVID-19. Before the pandemic, these cardiometabolic diseases already represented a substantial burden worldwide but now they are even more of a challenge.

Unité d'Information Médicale, Hôpitaux Universitaires Paris Centre, AP-HP, Paris, France

6 Digestive Surgery Unit, Centre Hospitalier Universitaire de Guadeloupe, Pointe-à-Pitre, Guadeloupe

7 DRCI-URC Eco Ile-de-France (AP-HP), Assistance Publique-Hôpitaux de Paris, Paris, France

8 Assistance Publique-Hôpitaux de Paris, Service de Santé Publique, Henri Mondor-Albert- Chenevier, Créteil, France

9 Thoracic Surgery Department, Cochin Hospital, APHP Centre, University of Paris, Paris, France

10 INSERM U1138 Team «Cancer, Immune Control, and Escape», Cordeliers Research Center, University of Paris, Paris, France 
Diabetes is known to increase the susceptibility to acute respiratory infections and, with obesity, they represent independent risk factors for severe pneumonia [1,2]. Although obesity has been reported to offer protection in the setting of acute respiratory distress syndrome [3], several studies have associated it with adverse outcomes among individuals with SARS-CoV-2 infection. The Lille Intensive Care COVID-19 and Obesity study group reported a sevenfold increase in the risk of mechanical ventilation in individuals with a body mass index $(\mathrm{BMI})>35 \mathrm{~kg} / \mathrm{m}^{2}$ admitted with COVID-19 compared with those with a BMI $<25$ [4]. In New York City, among individuals with COVID-19 under 60 years of age, having a BMI between 30 and 34.9 and $>35$ was associated with a 1.8- and 3.6-fold higher risk of being admitted to critical care, respectively [5]. Furthermore, the increased prevalence of obesity in older individuals in Italy compared with China has been pointed out as a potential explanation for the differences in mortality that have been reported between the two countries [6]. Several hypotheses have been made to explain the association between obesity and adverse outcomes in individuals with COVID-19. From a mechanical standpoint, obese individuals have a decreased expiratory reserve volume, a decreased functional capacity, and a lower total respiratory system compliance. Additionally, in these patients, the respiratory function is diminished in the supine position [7]. Moreover, the increased secretion of cytokines in excess adipose tissue has also been claimed to participate in the higher morbidity, through the impairment of immune responses [8] and their effects on lung parenchyma and bronchi $[9,10]$. Lastly, obesity drives an increased risk of chronic diseases that are associated with a severe outcome in COVID-19 [11-13].

The prevalence of obesity has increased exponentially in the last decades with over half of the European population now classified as overweight or obese [14]. In France, the prevalence of obesity in 2016 was estimated to be $21.6 \%$ (17.4-25.9) by the World Health Organization [15].

Bariatric surgery (BS) has been shown to be the only effective mean leading to sustained weight loss [16] and remission or improvement of obesity-related comorbidities [17, 18]. Several reports have proposed that BS has a protective effect against the onset of chronic diseases such as type 2 diabetes [19] as well as against some cancers [20, 21]. For these reasons, BS has obtained deep dissemination worldwide. For instance, in France, more than 500,000 individuals had BS before 2018 [22].

Based on these data, we made the hypothesis that a history of BS could have a protective effect against the occurrence of the severe form of COVID-19 and its related death. We used the French National Health Insurance Information System [23] to compare the need for invasive mechanical ventilation and death in obese individuals hospitalized for COVID-19 with or without a history of BS.

\section{Materials and Methods}

\section{Design and Study Population}

This study is based on exhaustive data from the "Programme de Médicalisation des Systèmes d'Information" database (Program for Medicalization of Information Systems, PMSI) of the national health care system. This database collects data on all hospital stays, regardless of their duration, in French public and private hospitals. In addition to its administrative purpose (monitoring of hospital activity based on the Diagnostic-Related Groups (DRGs) model, informing pricing and strategic decisions by payers and policy-makers), the database is also used for epidemiological purposes in several areas [21, 24].

Data in PMSI are collected on a real-time basis and include the ciphered identification of each patient, the main diagnosis leading to the hospital stay, the associated comorbidities, and the potential complications during their stay, as well as additional administrative and medical information, including treatment modalities. Data are abstracted by medical doctors and certified reviewers. The recording of the diagnosis relies on the last version of the International Classification of Diseases (ICD, World Health Organization). The recording of medical and surgical procedures is based on the French "Classification Commune des Actes Médicaux" (Common Classification of Medical Procedures, CCAM). Institutions are audited annually by the national health insurance services to verify the accuracy of their data, in a random selection process. Since the financial funds of all institutions rely on their declared data, the exhaustive coding can generate higher fees.

A two-step procedure was performed. First, we constituted a retrospective working cohort study including all the patients hospitalized for morbid obesity (ICD codes E66.-00 to E66.09, E66.-20 to E66.-29, E66.-80 to E66.-89, and E66.-90 to E66.-99) and discharged between January 1, 2010, and December 31, 2019. In this retrospective cohort study, BS stays were defined as those containing at least one of the most commonly performed bariatric procedures in France including open (HFMA009, HFMA006) and laparoscopic (HFMC007, HFMC005) adjustable gastric banding, open (HFCA001) and laparoscopic (HFCC003) gastric bypass, and open (HFFA011) and laparoscopic (HFFC018) sleeve gastrectomy.

Second, we identified among these patients, those aged 1575 years admitted for a COVID-19 (ICD-10 code U07.1* [* in $0,1,2,4,5])$ and discharged between January 1,2020 , and May 15, 2020. The final cohort study is composed of two groups: operated (BS) and non-operated (non-BS). Queries were carried out on June 2, 2020.

Data were anonymously collected and analyzed. Because of the retrospective analysis on an anonymous database, no informed consent was possible, and Institutional Review Board (IRB) approval was unnecessary according to French laws on biomedical research. 
We determined a priori the following baseline data to be extracted for each COVID-19 patient: age, sex, hypertension (ICD-10 code I10), diabetes (ICD-10 codes E10.-, E11.-, E12.-, E13.-, E14.-), chronic obstructive pulmonary disease (COPD; ICD-10 codes J44.-), cardiac failure (ICD-10 codes I50.-), and active cancer (ICD-10 codes C-), as well as the two main outcome variables: death and invasive mechanical ventilation (CCAM codes: GLLD004, GLLD006, GLLD008, GLLD013, GLLD015). Additional comorbidities needed to calculate the Charlson Comorbidity Index (CCI) were also extracted (Appendix) [25]. BMI classes (30-39.9; 40-50; > 50) were retrieved when available.

\section{Statistics}

Continuous variables were presented as the means and standard deviation (SD), and categorical variables were summarized as numbers and percentages. Differences between groups were analyzed using the Student's $t$ test and Pearson's $\chi^{2}$ test for continuous and categorical variables, respectively. Univariate analysis was used to assess the characteristics associated with mortality and need for invasive mechanical ventilation in the whole population and in subgroups. Different logistic regression models implying backward elimination of non-significant variables were then built by entering factors associated with the need for invasive mechanical ventilation or mortality at univariate analysis, to assess the factors independently associated with these outcome parameters in the whole population. As baseline BMI was not available in all the patients (differently from all the other variables), we built models including or not including this variable. Because of the expected significant interaction, we did not enter $\mathrm{CCI}$ in the multivariable methods but preferred entering relevant clinical variables. For these variables, collinearity was checked in both models with the variance inflation factor (VIF). The predefinition of a limited number of clinically relevant variables to be retrieved from the national database prevented overfitting.

Furthermore, exact matching (by sex; age classes 15-30, $31-45,46-60,61-75$ years; $\mathrm{BMI} \geq 40$ or not; and $\mathrm{CCI} \leq 3$ or not) was generated randomly to match subjects in the BS and non-BS group. Two random generation of pairs were performed.

For each test, a $p$ value $<0.05$ was considered statistically significant. $\mathrm{R}$ version 4.0.2 was used for statistical analysis.

\section{Results}

This retrospective, population-based, multi-institutional, cohort study based on the French electronic health database included 4,248,253 obese individuals, aged 15 to 75 years (Fig. 1). All inpatients, with a diagnosis of obesity recorded during a hospital stay between 2010 and 2019, were followed up for a mean $(\mathrm{SD})$ of 5.22 years $( \pm 2.93)$ for the non-BS group and 5.43 years $( \pm 2.45)$ for the BS group. Among $4,248,253$ obese individuals, $8286(0.2 \%)$ were hospitalized between January 1 and May 15, 2020, with the diagnosis of COVID-19. A mean of 1.22 stay per patient was recorded for a total of 10,069 COVID-19 stays. These 8226 individuals met the selection criteria to constitute the study cohort (Fig. 1). Of note, during the same period of time, 78,995 additional individuals $(0.12 \%$ of the French population), with no history of previous hospitalization for obesity, were hospitalized in France with a diagnosis of COVID-19.

Fifty-four percent of individuals were aged 61 to $75,23 \%$ were men, $7 \%$ had COPD, $6.9 \%$ had heart failure, $7.3 \%$ had a history of cancer, $35.2 \%$ were diabetic, and $40.2 \%$ had hypertension. Information on BMI was available in 7208 individuals $(87.3 \%$ ). BMI was between 30 and 39.9 in $78.6 \%$, between 40 and 50 in $18.3 \%$ and above 50 in $3.1 \%$ of individuals, and the mean CCI score was 3.15. A total of 541 patients (6.6\%) had a history of BS between 2010 and 2019 (BS group), and the remaining patients ( $n=7685 ; 93.4 \%)$ constitute the non-BS group (Table 1). As expected, individuals in the non-BS group were significantly older and more frequently male and had a higher mean CCI score, COPD more frequently, heart failure, history of cancer, diabetes, and hypertension. There were more individuals with BMI class 40-50 among the BS group while the BMI of the individuals of nonBS group was predominantly under 40 (Table 1).

Invasive mechanical ventilation was necessary in 1196 (14.54\%) patients and death occurred in 1117 (13.58\%) subjects in the whole population (Table 1).

Univariate analysis indicated that age, male sex, higher CCI score, COPD, cardiac failure, history of cancer, type 2 diabetes, and hypertension were significantly associated with an increased risk of death; all but COPD were associated with an increased risk of invasive mechanical ventilation in the whole study population (Table 2). BS had a protective effect because $7 \%$ of individuals in the BS group versus $15 \%$ of those in the non-BS group required invasive mechanical ventilation $(p<0.0001)$ and $3.5 \%$ in the BS subpopulation versus $14.2 \%$ of those in the non-BS subpopulation died $(p<0.0001)$. Among patients with a baseline BMI between 30 and 39.9, the need for mechanical ventilation occurred in $15 / 226(6.6 \%)$ and in $827 / 5443(15.2 \%)$ of those who had or had not undergone BS ( $p=0.00039)$, respectively, in the time frame between initial management of obesity and hospitalization for COVID-19. These figures were 21/274 (7.7\%) and $165 / 1049(15.7 \%)(p=0.00063)$ when baseline BMI was between 40 and 50, and 2/41 (2.4\%) and 19/175 (16\%) $(p=\mathrm{N} /$ A) when baseline BMI was more than 50 in BS or non-BS patients, respectively.

Among patients with baseline BMI between 30 and 39.9, mortality was $7 / 226(3.1 \%)$ and $773 / 5443(14.2 \%)$ in those who had or had not undergone BS $(p=0.0000021)$. These 
Fig. 1 Flowchart of the study population

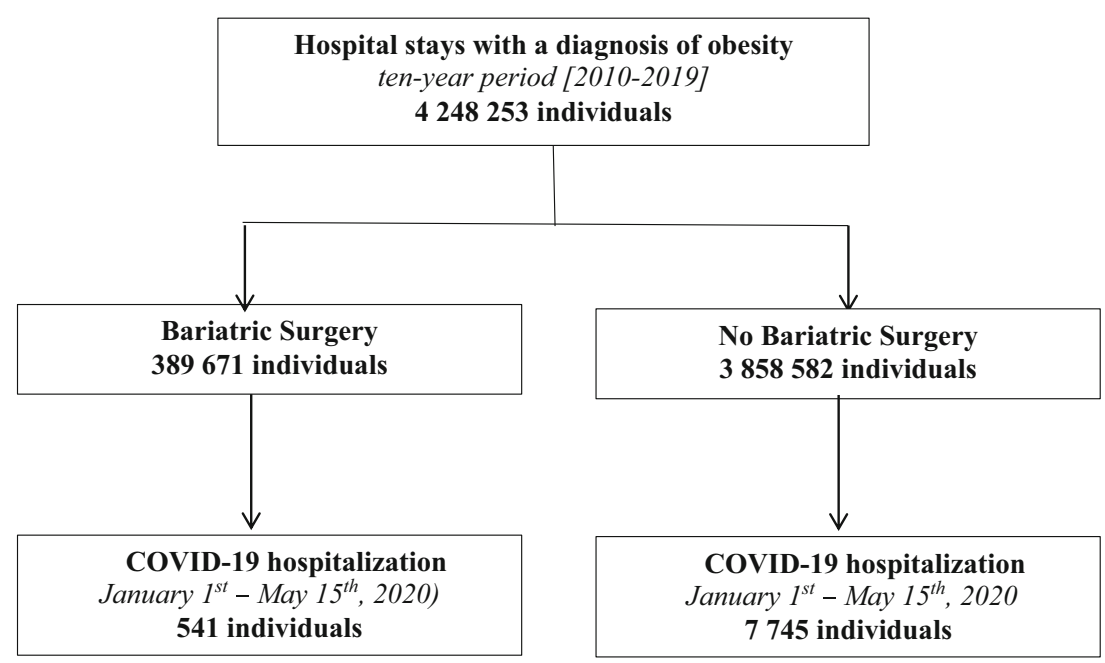

figures were $11 / 274(4.0 \%)$ and $169 / 1049(16.1 \%)(p=$ 0.00000020) when baseline BMI was between 40 and 50 and $1 / 41(2.4 \%)$ and 28/175 (16\%) $(p=\mathrm{N} / \mathrm{A})$ when baseline BMI was more than 50 in BS or non-BS patients, respectively.

Table 1 Univariate analysis of baseline risk factors distribution in COVID-19 obese patients in BS and NBS cohorts

\begin{tabular}{|c|c|c|c|c|}
\hline \multirow[b]{2}{*}{ Characteristic } & \multicolumn{2}{|l|}{ No. $(\%)$ of patients } & \multirow[b]{2}{*}{$\begin{array}{l}\text { No bariatric surgery } \\
n=7745\end{array}$} & \multirow[b]{2}{*}{$p$ value } \\
\hline & $\begin{array}{l}\text { Study population } \\
n=8286\end{array}$ & $\begin{array}{l}\text { Bariatric surgery } \\
n=541\end{array}$ & & \\
\hline Age, mean (SD), year & $59.1(12.6)$ & $49.8(12.0)$ & $59.8(12.4)$ & $<0.0001$ \\
\hline $34-45$ & $251(3)$ & $33(6.1)$ & $218(2.8)$ & $<0.0001$ \\
\hline $15-30$ & $1057(12.8)$ & $158(29.2)$ & $899(11.6)$ & $<0.0001$ \\
\hline $46-60$ & $2470(29.8)$ & $239(44.2)$ & $2231(28.8)$ & $<0.0001$ \\
\hline $60-75$ & $4508(54.4)$ & $111(20.5)$ & $4397(56.8)$ & $<0.0001$ \\
\hline Sex, M & $4296(51.8)$ & $127(23.5)$ & $4169(53.8)$ & $<0.0001$ \\
\hline Sex, F & $3990(48.2)$ & $414(76.5)$ & $3576(46.2)$ & \\
\hline $\mathrm{BMI}(n=7208)$ & & & & $<0.0001$ \\
\hline $30-39.9$ & 5669 (78.6) & $226(41.8)$ & 5443 (81.6) & \\
\hline $40-50$ & $1323(18.3)$ & $274(50.6)$ & 1049 (15.7) & \\
\hline$>50$ & $216(3.1)$ & $41(7.6)$ & $175(2.7)$ & \\
\hline $\mathrm{CCI}$ & $3.15(2.58)$ & $1.451(1.83)$ & $3.26(2.58)$ & $<0.0001$ \\
\hline COPD, yes & $583(7)$ & $16(3)$ & $567(7.3)$ & $<0.0001$ \\
\hline COPD, no & 7703 (93) & $525(97)$ & $7178(92.7)$ & \\
\hline Cardiac failure, yes & $569(6.9)$ & $18(3.3)$ & $551(7.1)$ & 0.0008 \\
\hline Cardiac failure, no & 7717 (93.1) & $523(96.7)$ & $7194(92.9)$ & \\
\hline Cancer, yes & $608(7.3)$ & $11(2)$ & $597(7.7)$ & $<0.0001$ \\
\hline Cancer, no & $7678(92.7)$ & $530(98)$ & $7148(92.3)$ & \\
\hline Diabetes, yes & $2917(35.2)$ & $66(12.2)$ & $2851(36.8)$ & $<0.0001$ \\
\hline Diabetes, no & $5369(64.8)$ & $475(87.8)$ & $4894(63.2)$ & \\
\hline Hypertension, yes & $3331(40.2)$ & $109(20.2)$ & $3222(41.6)$ & $<0.0001$ \\
\hline Hypertension, no & $4955(59.8)$ & $432(78.9)$ & $4523(58.4)$ & \\
\hline Invasive mechanical ventilation, yes & $1196(14.4)$ & $38(7)$ & $1158(15)$ & $<0.0001$ \\
\hline Invasive mechanical ventilation, no & $7090(85.6)$ & $503(93)$ & $6587(85)$ & \\
\hline Death, yes & $1117(13.5)$ & $19(3.5)$ & $1098(14.2)$ & $<0.0001$ \\
\hline Death, no & $7169(86.5)$ & $522(96.5)$ & $6647(85.8)$ & \\
\hline
\end{tabular}

CCI, Charlson Comorbidity Index

$C O P D$, chronic obstructive pulmonary disease 
Table 2 Univariate analysis of baseline risk factors for invasive mechanical ventilation and death in COVID-19 obese patients

\begin{tabular}{|c|c|c|c|c|c|c|}
\hline \multirow[b]{2}{*}{ Characteristic } & \multicolumn{6}{|l|}{ No. $(\%)$ of patients } \\
\hline & $\begin{array}{l}\text { Invasive mechanical } \\
\text { ventilation } n=1196\end{array}$ & $\begin{array}{l}\text { No invasive mechanical } \\
\text { ventilation } n=7090\end{array}$ & $p$ value & Death $n=1117$ & Alive $n=7169$ & $p$ value \\
\hline Age, mean (SD), y & $61.8(9.9)$ & $58.7(13.0)$ & $<0.0001$ & $66.6(7.7)$ & $57.9(12.8)$ & $<0.0001$ \\
\hline $15-30$ & $6(0.5)$ & $245(3.5)$ & $<0.0001$ & $3(0.3)$ & $248(3.5)$ & $<0.0001$ \\
\hline $31-45$ & $79(6.6)$ & $978(13.8)$ & $<0.0001$ & $19(1.7)$ & $1038(14.5)$ & $<0.0001$ \\
\hline $46-60$ & $367(30.7)$ & $2103(29.7)$ & .4738 & $161(14.4)$ & 2309 (32.2) & $<0.0001$ \\
\hline $60-75$ & $744(62.2)$ & $3764(53.1)$ & $<0.0001$ & 934 (83.6) & 3574 (49.9) & $<0.0001$ \\
\hline Sex, M & $793(66.3)$ & $3503(49.4)$ & $<0.0001$ & $737(66)$ & 3559 (49.6) & $<0.0001$ \\
\hline Sex, F & $403(33.7)$ & 3587 (50.6) & & $380(34)$ & $3610(50.4)$ & \\
\hline BMI $(n=7208)$ & & & 0.092 & & & 0.98 \\
\hline $30-39.9$ & $842(80.3)$ & $4827(78.4)$ & & $780(78.9)$ & 4889 (78.6) & \\
\hline $40-50$ & $186(17.7)$ & $1137(18.5)$ & & $180(18.2)$ & $1143(18.4)$ & \\
\hline$>50$ & $21(2.0)$ & $195(3.1)$ & & $29(2.9)$ & $187(3.0)$ & \\
\hline $\mathrm{CCI}$ & $3.66(2.37)$ & $3.06(2.6)$ & $<0.0001$ & $5.13(2.92)$ & $2.84(2.38)$ & $<0.0001$ \\
\hline COPD, yes & $\begin{array}{l}94(7.9) \\
1102(92.1)\end{array}$ & $\begin{array}{l}489(6.9) \\
6601(93.1)\end{array}$ & .2286 & $\begin{array}{l}112(10) \\
1005(90)\end{array}$ & $\begin{array}{l}471(6.6) \\
6698(93.4)\end{array}$ & $<0.0001$ \\
\hline Cardiac failure, yes & $113(9.5)$ & $456(6.4)$ & .0001 & 144 (12.9) & $425(5.9)$ & $<0.0001$ \\
\hline Cardiac failure, no & 1083 (90.6) & 6634 (93.6) & & $973(87.1)$ & $6744(94.1)$ & \\
\hline Cancer, yes & $73(6.1)$ & $535(7.6)$ & $<0.0001$ & $197(17.6)$ & $411(5.7)$ & $<0.0001$ \\
\hline Cancer, no & $1123(93.9)$ & $6555(92.5)$ & & $920(82.4)$ & $6758(94.3)$ & \\
\hline Diabetes, yes & $545(45.6)$ & $2372(33.5)$ & $<0.0001$ & $531(47.5)$ & $2386(33.3)$ & $<0.0001$ \\
\hline Diabetes, no & $651(56.3)$ & $4718(66.5)$ & & $586(52.5)$ & $4783(66.7)$ & \\
\hline Hypertension, yes & $718(60)$ & $2613(36.9)$ & $<0.0001$ & $541(48.4)$ & $2790(38.9)$ & $<0.0001$ \\
\hline Hypertension, no & $478(40)$ & $4477(63.2)$ & & $576(51.6)$ & $4379(61.1)$ & \\
\hline Bariatric Surgery, yes & $38(3.2)$ & $503(7.1)$ & $<0.0001$ & $19(1.7)$ & $522(7.3)$ & $<0.0001$ \\
\hline Bariatric Surgery, no & $1158(96.8)$ & 6587 (92.9) & & $1098(98.3)$ & 6647 (92.7) & \\
\hline
\end{tabular}

$C O P D$, chronic obstructive pulmonary disease

CCI, Charlson Comorbidity Index

The analysis of preoperative factors influencing the need for mechanical ventilation (Tables 3 and 4) and mortality (Tables 5 and 6) was investigated in the multivariable analysis with or without BMI and the protective effect of BS was confirmed in both cases. The logistic regression (including sex, hypertension, age range, diabetes, cardiac failure, and

Table 3 Multivariate analysis of baseline risk factors for invasive mechanical ventilation. Model including the whole population

\begin{tabular}{llll}
\hline Characteristic & OR & $95 \%$ CI & $p$ value \\
\hline Age, mean (SD), year & & & $<0.0001$ \\
$15-30$ & Reference & & \\
$31-45$ & 1.17 & $1.06-1.28$ & \\
$46-60$ & 1.36 & $1.13-1.64$ & \\
$60-75$ & 1.59 & $1.20-2.10$ & \\
Sex, M & 1.76 & $1.54-2.00$ & $<0.0001$ \\
Hypertension & 2.25 & $1.97-2.56$ & $<0.0001$ \\
Bariatric surgery & 0.67 & $0.48-0.95$ & 0.025 \\
\hline
\end{tabular}

BS) showed that the risk of invasive mechanical ventilation increased with age, being higher in the age class $61-75$ years (OR 1.59; 95\% CI 1.20-2.10; $p<0.0001$ ), in male sex (OR 1.76 ; $95 \%$ CI $1.54-2.00 ; p<0.0001)$, and in those with hypertension (OR 2.25; 95\% CI 1.97-2.56; $p<0.0001$ ), whereas

Table 4 Multivariate analysis of baseline risk factors for invasive mechanical ventilation. Model including the 7208 patients whose baseline BMI class was available

\begin{tabular}{llll}
\hline Characteristic & OR & $95 \%$ CI & $p$ Value \\
\hline Age, mean (SD), year & & & 0.00042 \\
$15-30$ & Reference & & \\
$31-45$ & 1.18 & $1.08-1.30$ & \\
$46-60$ & 1.40 & $1.16-1.69$ & \\
$60-75$ & 1.65 & $1.25-2.19$ & \\
Sex, M & 1.77 & $1.55-2.02$ & $<0.0001$ \\
Hypertension & 2.25 & $1.97-2.56$ & $<.0001$ \\
Bariatric surgery & 0.67 & $0.47-0.94$ & 0.020 \\
\hline
\end{tabular}


Table 5 Multivariate analysis of baseline risk factors for death. Model including the whole population

Death

\begin{tabular}{llll}
\hline Characteristic & OR & $95 \%$ CI & $p$ value \\
\hline Age, mean (SD), year & & & $<0.0001$ \\
$15-30$ & Reference & & \\
$31-45$ & 3.10 & $2.69-3.58$ & \\
$46-60$ & 9.63 & $7.25-12.79$ & \\
$60-75$ & 29.87 & $19.52-45.73$ & \\
Sex, M & 1.48 & $1.28-1.69$ & $<0.0001$ \\
Cardiac failure & 1.53 & $1.24-1.89$ & $<0.0001$ \\
Cancer & 2.81 & $2.32-3.41$ & $<0.0001$ \\
Diabetes & 1.33 & $1.16-1.52$ & $<0.0001$ \\
Bariatric surgery & 0.50 & $0.31-0.80$ & 0.0039 \\
\hline
\end{tabular}

BS showed an independent protective effect (OR 0.67; 95\% CI $0.48-0.95 ; p<0.025$ ).

Concerning the risk of death, logistic regression (including sex, hypertension, classes of age, diabetes, cardiac failure, cancer, COPD, and BS) showed that older age was strongly associated with COVID-19-related death with an OR of 29.87 (95\% CI 19.52-45.73) in the age range $61-75$ years $(p<0.0001)$. The risk of mortality was also independently associated with male sex (OR 1.48; 95\% CI 1.28-1.69), known history of heart failure (OR 1.53 ; 95\% CI 1.24-1.89; $p<0.0001$ ), cancer (OR 2.81; 95\% CI 2.32-3.41; $p<0.0001$ ), and diabetes (OR $1.33 ; 95 \%$ CI $1.16-1.52 ; p<0.0001)$ whereas BS showed a protective effect (OR $0.450 ; 95 \%$ CI $0.31-$

Table 6 Multivariate analysis of baseline risk factors for death. Model including the 7208 patients for whom the baseline BMI class was available

\begin{tabular}{llll}
\hline Characteristic & OR & $95 \%$ CI & $p$ Value \\
\hline Age, mean (SD), year & & & $<0.0001$ \\
$15-30$ & Reference & & \\
$31-45$ & 3.12 & $2.70-3.59$ & \\
$46-60$ & 9.71 & $7.31-12.90$ & \\
60-75 & 30.25 & $19.76-46.33$ & \\
Sex, M & 1.52 & $1.32-1.75$ & $<0.0001$ \\
Cardiac failure & 1.52 & $1.23-1.87$ & $<0.0001$ \\
Cancer & 2.83 & $2.33-3.42$ & $<0.0001$ \\
Diabetes & 1.32 & $1.15-1.51$ & $<0.0001$ \\
Bariatric surgery & 0.44 & $0.27-0.71$ & 0.00086 \\
BMI & & & 0.0018 \\
30-39.9 & Reference & & \\
$40-50$ & 1.26 & $1.09-1.46$ & \\
$>50$ & 1.59 & $1.19-2.12$ & \\
\hline
\end{tabular}

$0.80 ; p=0.0039)$. When BMI classes were included in specific models for the 7208 individuals for whom information was available, increasing BMI was significantly associated with the risk of death (BMI class 40-50, OR 1.26; 95\% CI 1.091.46; BMI class > 50 (OR 1.59; 95\% CI 1.19-2.12).

Two random exact matching tests considering 243 subjects in the BS group indicate that BS was still associated with a protective effect against the risk of mechanical ventilation and death (Table 7).

\section{Discussion}

In this French medico-administrative nationwide study, the history of BS was independently associated with a significant reduction in the risk of mortality in obese individuals developing COVID-19 (OR 0.50; 95\% CI 0.31-0.80; $p<0.0039$ ). BS was also independently associated with a reduced risk of invasive mechanical ventilation (OR 0.67; 95\% CI 0.48-0.95; $p=0.025)$. Ventilation was required by $15 \%$ of the BS group compared with $7 \%$ of the non-BS group $(p<0.0001)$.

As the epidemic spreads around the world, scientists have raced to identify risk factors for severe forms of COVID-19 and death because the outbreak has challenged the health care systems of all countries affected by the epidemic. Individuals at risk have more chances not only to become infected but also to develop severe forms of the disease including the need for mechanical ventilation and death. Given the shortage of resources, governments must warn more vulnerable individuals to be stringent in respecting social distancing measures [26]. For these reasons, high-quality data on all potential risk factors are needed to identify vulnerable individuals and help governments to draw reliable guidance to minimize social and economic disruption linked to restriction measures [27].

In initial studies, older age, male sex, hypertension, diabetes, cardiovascular disease, and malignancy have been reported as risk factors for severe COVID-19 and death [11-13], while no emphasis was put on increased BMI as a risk factor [28-30].

A French study then showed that disease severity increases with BMI and that the need for invasive mechanical ventilation is associated with severe obesity (BMI $>35$ ) regardless of age, sex, diabetes, and hypertension [4]. These data were confirmed in another French study reporting that obese individuals had higher odds of developing severe COVID-19 than individuals without obesity (adjusted ORs ranged between 1.80 and 2.03) [31]. Awareness of obesity as a main risk factor for severe COVID-19 outcome led the French Ministry of Solidarity and Health to produce a "care sheet for those in a situation of obesity during the COVID-19 epidemic" and general practitioners were authorized to provide work stoppages for obese individuals [32]. 
Table 7 Univariate analysis of invasive mechanical ventilation and death for two random matched BS and NBS groups

\begin{tabular}{|c|c|c|c|c|c|c|c|c|c|}
\hline Characteristics & NBS & BS & $N$ of patients & $p$ & & NBS & BS & $N$ of patients & $p$ \\
\hline \multicolumn{10}{|l|}{ 1st random matched group } \\
\hline $\begin{array}{l}\text { Invasive mechanical ventilation, no } \\
\text { Invasive mechanical ventilation, yes }\end{array}$ & $\begin{array}{l}207 \\
36\end{array}$ & $\begin{array}{l}227 \\
16\end{array}$ & $\begin{array}{l}434 \\
52\end{array}$ & $<0.05$ & $\begin{array}{l}\text { Death, no } \\
\text { Death, yes }\end{array}$ & $\begin{array}{l}225 \\
18\end{array}$ & $\begin{array}{l}237 \\
6\end{array}$ & $\begin{array}{l}462 \\
24\end{array}$ & $<0.05$ \\
\hline$N$ of patients & 243 & 243 & 486 & & $N$ of patients & 243 & 243 & 486 & \\
\hline \multicolumn{10}{|l|}{ 2nd random matched group } \\
\hline $\begin{array}{l}\text { Invasive mechanical ventilation, no } \\
\text { Invasive mechanical ventilation, yes }\end{array}$ & $\begin{array}{l}207 \\
36\end{array}$ & $\begin{array}{l}227 \\
16\end{array}$ & $\begin{array}{l}434 \\
52\end{array}$ & $<0.05$ & $\begin{array}{l}\text { Death, no } \\
\text { Death, yes }\end{array}$ & $\begin{array}{l}225 \\
18\end{array}$ & $\begin{array}{l}237 \\
6\end{array}$ & $\begin{array}{l}462 \\
24\end{array}$ & $<0.05$ \\
\hline$N$ of patients & 243 & 243 & 486 & & $N$ of patients & 243 & 243 & 486 & \\
\hline
\end{tabular}

$N B S$, no bariatric surgery; $B S$, bariatric surgery

BS has developed widely in the last two decades in concomitance with the sharp increase in the prevalence of obesity worldwide [33]. There is a large body of evidence demonstrating that BS is effective in achieving a sustained weight loss, maintained in the long term [17]. BS-induced weight loss is also effective in reversing the metabolic comorbidities linked to obesity, such as diabetes, which stands among the most common risk factors for a severe outcome in COVID-19, as discussed above [19, 34]. For these reasons, we made the hypothesis that a history of BS could have a protective effect against mortality and the need for invasive mechanical ventilation in obese individuals. Interestingly, this study confirmed the findings of previous studies indicating that older age, male sex, malignancy, heart failure, and diabetes are independent risk factors for increased mortality, while hypertension was no longer significantly associated with mortality in multivariable analysis.

Other mechanisms that may account for the protective effect of BS include the improvement of respiratory function that occurs with the loss of weight. Finally, the reversal of low-grade systemic inflammation linked to obesity due to BS-induced loss of weight may also play an important role in reducing the risk of severe COVID-19 [35]. All these factors may account for the significantly lower mortality and need for invasive mechanical ventilation in obese individuals with a history of BS compared to those with no history of BS.

The present study carries some weaknesses linked to the retrospective study design, the potentially missing data on COVID-19 code, which has been created ad hoc at the beginning of the epidemic, and the lack of information on BMI for $12.7 \%$ (1048 out of 8256 ) of the individuals included. Furthermore, the BS group included more women and younger individuals with a lower CCI score than the non-BS control group. However, while half of the individuals in the BS group were in the 40-50 BMI class, less than one in six in the non-BS group were in this class. Indeed, despite of differences in the characteristics of the BS and non-BS groups, the independent protective effects of BS on respiratory failure and death are clearly shown by multivariable analysis when the different classes of BMI were included in the analysis. These results were confirmed by the random generated exact matching analysis by sex, age, BMI, and CCI that was done to neutralize any bias linked to the baseline characteristics of patients.

The PMSI database has been largely used in the past for epidemiological studies and stands among the largest health databases in the world. Finally, our analysis confirmed data reported in previous studies on risk factors for COVID-19related death suggesting the reliability of our findings.

\section{Conclusion}

Despite its retrospective design, this study is the first to report the protective effect of BS against the severity of COVID-19. This finding provides interesting information from an epidemiologic point of view and provides insights into the pathophysiology of COVID-19, especially in cases of severe presentations. To conclude, finding protective factors against COVID-19 could help in narrowing the population at risk. In doing so, preventative measures such as social distancing or serological screening could be targeted with more precision.

Authors' Contribution All authors have contributed equally to this work.

\section{Compliance with Ethical Standards}

Conflict of Interest The authors declare that they have no conflict of interest.

Ethical Approval Statement All procedures performed in studies involving human participants were in accordance with the ethical standards of the institutional and/or national research committee and with the 1964 Helsinki declaration and its later amendments or comparable ethical standards.

Informed Consent Statement Informed consent does not apply in this study. 


\section{Appendix}

Table 8 ICD-10 coding algorithms for Charlson comorbidities

ICD-10 coding algorithms for Charlson CIM10 code1

comorbidities

\begin{tabular}{|c|c|c|}
\hline Myocardial infarction & $\mathrm{I} 21 . * ; \mathrm{I} 22 . * ; \mathrm{I} 25.2$ & $\mathrm{I} 21$ * $^{*}$ \\
\hline Congestive heart failure & I09.9; I11.0; I13.0; I13.2; I25.5; I42.0; I42.5-I42.9; I43.*; I50.*; P29.0 & I09.9 \\
\hline Peripheral vascular disease & $\begin{array}{l}\text { I70.*; I71.*; I73.1; I73.8; I73.9; I77.1; I79.0; I79.2; K55.1; K55.8; K55.9; Z95.8; } \\
\quad \text { Z95.9 }\end{array}$ & $\mathrm{I} 70 . *$ \\
\hline Cerebrovascular disease & G45.*; G46.*; H34.0; I60.*-I69.* & G45.* \\
\hline Dementia & F00.*-F03.*; F05.1; G30.*; G31.1 & F00.*-F03.* \\
\hline Chronic pulmonary disease & $\mathrm{I} 27.8 ; \mathrm{I} 27.9 ; \mathrm{J} 40 . *-\mathrm{J} 47 . * ; \mathrm{J} 60 . *-\mathrm{J} 67 . * ; \mathrm{J} 68.4 ; \mathrm{J} 70.1 ; \mathrm{J} 70.3$ & $\mathrm{I} 27.8$ \\
\hline Rheumatic disease & M05.*; M06.*; M31.5; M32.*-M34.*; M35.1; M35.3; M36.0 & M05.* \\
\hline Peptic ulcer disease & $\mathrm{K} 25 . *-\mathrm{K} 28{ }^{*}$ & $\mathrm{~K} 25 . *-\mathrm{K} 28 *^{*}$ \\
\hline Mild liver disease & $\begin{array}{l}\text { B18.*; K70.0-K70.3; K70.9; K71.3-K71.5; K71.7; K73.*; K74.*; K76.0; } \\
\text { K76.2-K76.4; K76.8; K76.9; Z94.4 }\end{array}$ & $\mathrm{B} 18 *^{*}$ \\
\hline Diabetes without chronic complication & $\begin{array}{l}\text { E10.0; E10.1; E10.6; E10.8; E10.9; E11.0; E11.1; E11.6; E11.8; E11.9; E12.0; } \\
\text { E12.1; E12.6; E12.8; E12.9; E13.0; E13.1; E13.6; E13.8; E13.9; E14.0; E14.1; } \\
\text { E14.6; E14.8; E14.9 }\end{array}$ & E10.0 \\
\hline Diabetes with chronic complication & $\begin{array}{l}\text { E10.2-E10.5; E10.7; E11.2-E11.5; E11.7; E12.2-E12.5; E12.7; E13.2-; E13.5; } \\
\text { E13.7; E14.2-E14.5; E14.7 }\end{array}$ & E10.2-E10.5 \\
\hline Hemiplegia or paraplegia & G04.1; G11.4; G80.1; G80.2; G81.*; G82.*; G83.0-G83.4; G83.9 & G04.1 \\
\hline Renal disease & $\begin{array}{l}\text { I12.0; I13.1; N03.2-N03.7; N05.2-; N05.7; N18.*; N19.*; N25.0; Z49.0-; Z49.2; } \\
\quad \text { Z94.0; Z99.2 }\end{array}$ & $\mathrm{I} 12.0$ \\
\hline $\begin{array}{l}\text { Any malignancy, including lymphoma and } \\
\text { leukemia, except malignant neoplasm of skin }\end{array}$ & 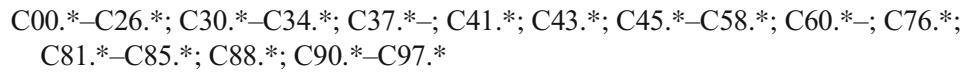 & $\mathrm{C} 00 *{ }^{*}-\mathrm{C} 26 .^{*}$ \\
\hline Moderate or severe liver disease & I85.0; I85.9; I86.4; I98.2; K70.4; K71.1; K72.1; K72.9; K76.5; K76.6; K76.7 & $\mathrm{I} 85.0$ \\
\hline Metastatic solid tumor & $\mathrm{C} 77 . * \mathrm{C} 80 . *$ & $\mathrm{C} 77 . *-\mathrm{C} 80 . *$ \\
\hline AIDS/HIV & $\mathrm{B} 20 . *-\mathrm{B} 22 . * ; \mathrm{B} 24 . *$ & $\mathrm{~B} 20 . *-\mathrm{B} 22 . *$ \\
\hline
\end{tabular}

\section{References}

1. Muller LMAJ, Gorter KJ, Hak E, et al. Increased risk of common infections in patients with type 1 and type 2 diabetes mellitus. Clin Infect Dis Off Publ Infect Dis Soc Am. 2005;41(3):281-8.

2. Luzi L, Radaelli MG. Influenza and obesity: its odd relationship and the lessons for COVID-19 pandemic. Acta Diabetol. 2020;57(6):759 64.

3. Ni Y-N, Luo J, Yu H, et al. Can body mass index predict clinical outcomes for patients with acute lung injury/acute respiratory distress syndrome? A meta-analysis. Crit Care Lond Engl. 2017;21(1):36.

4. Simonnet A, Chetboun M, Poissy J, et al. High prevalence of obesity in severe acute respiratory syndrome coronavirus-2 (SARS$\mathrm{CoV}-2$ ) requiring invasive mechanical ventilation. Obes Silver Spring Md. 2020;28(7):1195-9.

5. Lighter J, Phillips M, Hochman S, et al. Obesity in patients younger than 60 years is a risk factor for COVID-19 hospital admission. Clin Infect Dis Off Publ Infect Dis Soc Am. 2020;71(15):896-7.

6. Dietz W, Santos-Burgoa C. Obesity and its implications for COVID-19 mortality. Obes Silver Spring Md. 2020;28(6):1005.

7. Dixon AE, Peters U. The effect of obesity on lung function. Expert Rev Respir Med. 2018;12(9):755-67.
8. Huttunen R, Syrjänen J. Obesity and the risk and outcome of infection. Int J Obes 2005. 2013;37(3):333-40.

9. Zhang X, Zheng J, Zhang L, et al. Systemic inflammation mediates the detrimental effects of obesity on asthma control. Allergy Asthma Proc. 2018;39(1):43-50.

10. Jose RJ, Manuel A. Does coronavirus disease 2019 disprove the obesity paradox in acute respiratory distress syndrome? Obes Silver Spring Md. 2020;28(6):1007.

11. Huang C, Wang Y, Li X, et al. Clinical features of patients infected with 2019 novel coronavirus in Wuhan, China. Lancet Lond Engl. 2020;395(10223):497-506.

12. Zhou F, Yu T, Du R, et al. Clinical course and risk factors for mortality of adult inpatients with COVID-19 in Wuhan, China: a retrospective cohort study. Lancet Lond Engl. 2020;395(10229):1054-62.

13. Shi Y, Yu X, Zhao H, et al. Host susceptibility to severe COVID-19 and establishment of a host risk score: findings of 487 cases outside Wuhan. Crit Care Lond Engl. 2020;24(1):108.

14. Marques A, Peralta M, Naia A, et al. Prevalence of adult overweight and obesity in 20 European countries, 2014. Eur J Pub Health. 2018;28(2):295-300.

15. WHO | World Health Statistics 2017: Monitoring health for the SDGs. WHO. . http://www.who.int/gho/publications/world health_statistics/2017/en/ 
16. Maciejewski ML, Arterburn DE, Van Scoyoc L, et al. Bariatric surgery and long-term durability of weight loss. JAMA Surg. 2016;151(11):1046-55.

17. Chang S-H, Stoll CRT, Song J, et al. The effectiveness and risks of bariatric surgery: an updated systematic review and meta-analysis, 2003-2012. JAMA Surg. 2014;149(3):275-87.

18. Adams TD, Davidson LE, Litwin SE, et al. Weight and metabolic outcomes 12 years after gastric bypass. N Engl J Med. 2017;377(12):1143-55.

19. Carlsson LMS, Peltonen M, Ahlin S, et al. Bariatric surgery and prevention of type 2 diabetes in Swedish obese subjects. N Engl J Med. 2012;367(8):695-704.

20. Schauer DP, Feigelson HS, Koebnick C, et al. Bariatric surgery and the risk of cancer in a large multisite cohort. Ann Surg. 2019;269(1):95-101.

21. Bailly L, Fabre R, Pradier C, et al. Colorectal Cancer risk following bariatric surgery in a nationwide study of French individuals with obesity. JAMA Surg. 2020;155(5):395-402.

22. ameli.fr - Rapports Charges et produits pour les années 2018 à 2020. Accessed June 15, 2020. https://www.ameli.fr/l-assurancemaladie/statistiques-et-publications/rapports-et-periodiques/ rapports-charges-produits-de-1-assurance-maladie/rapportscharges-et-produits-pour-2018-a-2020/rapport-charges-et-produitspour-1-annee-2019.php

23. Scailteux L-M, Droitcourt C, Balusson F, et al. French administrative health care database (SNDS): the value of its enrichment. Therapie. 2019;74(2):215-23.

24. Bobbio A, Dechartres A, Bouam S, et al. Epidemiology of spontaneous pneumothorax: gender-related differences. Thorax. 2015;70(7):653-8.

25. Charlson ME, Pompei P, Ales KL, et al. A new method of classifying prognostic comorbidity in longitudinal studies: development and validation. J Chronic Dis. 1987;40(5):373-83.

26. [Withdrawn] Guidance on social distancing for everyone in the UK. GOV.UK. Accessed June 15, 2020. https://www.gov.uk/ government/publications/covid-19-guidance-on-social-distancing- and-for-vulnerable-people/guidance-on-social-distancing-foreveryone-in-the-uk-and-protecting-older-people-and-vulnerableadults

27. Jordan RE, Adab P, Cheng KK. Covid-19: risk factors for severe disease and death. BMJ. 2020;368:m1198.

28. Li Q, Guan X, Wu P, et al. Early transmission dynamics in Wuhan, China, of novel coronavirus-infected pneumonia. N Engl J Med. 2020;382(13):1199-207.

29. Grasselli G, Zangrillo A, Zanella A, et al. Baseline characteristics and outcomes of 1591 patients infected with SARS-CoV-2 admitted to ICUs of the Lombardy region, Italy. JAMA. 2020;323(16): 1574-81.

30. Bhatraju PK, Ghassemieh BJ, Nichols M, et al. Covid-19 in critically ill patients in the Seattle region - case series. N Engl J Med. 2020;382(21):2012-22.

31. Caussy C, Pattou F, Wallet F, et al. Prevalence of obesity among adult inpatients with COVID-19 in France. Lancet Diabetes Endocrinol. 2020;8(7):562-4.

32. DGOS_Michel.C, DGOS_Michel.C. Obésité et Covid-19. Ministère des Solidarités et de la Santé. Published June 15, 2020. Accessed June 15, 2020. http://solidarites-sante.gouv.fr/soins-etmaladies/prises-en-charge-specialisees/obesite/article/obesite-etcovid-19

33. Angrisani L, Santonicola A, Iovino P, et al. Bariatric surgery worldwide 2013. Obes Surg. 2015;25(10):1822-32.

34. Buchwald H, Estok R, Fahrbach K, et al. Weight and type 2 diabetes after bariatric surgery: systematic review and meta-analysis. Am J Med. 2009;122(3):248-256.e5.

35. Askarpour M, Khani D, Sheikhi A, et al. Effect of bariatric surgery on serum inflammatory factors of obese patients: a systematic review and meta-analysis. Obes Surg. 2019;29(8):2631-47.

Publisher's Note Springer Nature remains neutral with regard to jurisdictional claims in published maps and institutional affiliations. 